\title{
Carbapenemasas en Pseudomonas aeruginosa con susceptibilidad disminuida a los carbapenémicos después de una década, desde VIM a KPC
}

\section{Carbapenemases in Pseudomonas aeruginosa with decreased susceptibility to carbapenems after a decade: from VIM to KPC}

\author{
Margarita Enberg Gaete1, Marcela Puente Valenzuela', Aniela Wozniak Bachero², Claudia Castillo Vega ${ }^{3}$, \\ Nicolás Villagra Marín²,4, Jaime Labarca Labarca ${ }^{5,6}$ y Patricia García Cañete ${ }^{2,6}$
}

\author{
${ }^{1}$ Alumnas de Postgrado del Departamento de Enfermedades Infecciosas del Adulto, Escuela de Medicina. Pontificia Universidad Católica de Chile. Santiago, Chile. \\ 2Departamento de Laboratorios Clínicos. Escuela de Medicina. Pontificia Universidad Católica de Chile. Santiago, Chile. \\ ${ }^{3}$ Laboratorio de Microbiología. Servicio de Laboratorios Clínicos. Red de Salud UC-CHRISTUS. Santiago, Chile. \\ ${ }^{4}$ Escuela de Tecnología Médica, Facultad de Medicina, Universidad Andres Bello. Santiago, Chile. \\ ${ }^{5}$ Departamento de Enfermedades Infecciosas del Adulto, Escuela de Medicina. Pontificia Universidad Católica de Chile. Santiago, Chile. \\ ${ }^{6}$ Millennium Initiative for Collaborative Research on Bacterial Resistance (MICROB-R), Santiago, Chile.
}

Financiamiento: Fondos de Investigación, Departamento de Laboratorios Clínicos.

Sin conflictos de interés.

Recibido (segunda versión): 18 de junio de 2020 / Aceptado: 24 de junio de 2020

\section{Resumen}

Introducción: Pseudomonas aeruginosa es relevante en infecciones asociadas a la atención de salud, principalmente cuando presenta resistencia a carbapenémicos. Objetivos: Estudiar la producción de carbapenemasas en $P$. aeruginosa, con susceptibilidad disminuida a carbapenémicos procesadas en el Laboratorio de Microbiología de la Red de Salud UC-CHRISTUS entre 2014-2015, y compararlas con las cepas estudiadas en 2004-2005. Métodos: Entre enero de 2014 y junio de 2015, se aislaron 459 cepas de $P$. aeruginosa provenientes de muestras clínicas. La susceptibilidad fue determinada por dilución en agar y a las cepas con susceptibilidad disminuida a carbapenémicos se les realizó test de carbaNP. Las cepas positivas fueron estudiadas por RPC para genes $b l a_{\mathrm{VIM}}, b l a_{\mathrm{VIM}-1}, b l a_{\mathrm{VIM}-2}, b l a_{\mathrm{IMP}}, b l a_{\mathrm{NDM}}, b l a_{\mathrm{KPC}}, b l a_{\mathrm{OXA}}$ y $b l a_{\mathrm{IMI}}$. Se realizó en cepas seleccionadas electroforesis de campo pulsado. Resultados: De las 459 cepas estudiadas, 300 presentaban susceptibilidad disminuida a carbapenémicos (65,3\%). De éstas, 183 fueron viables para estudio, correspondientes a 164 pacientes. El test de carbaNP fue positivo en 44 cepas de las 183 cepas (24\%). Los genes de resistencia encontrados fueron: $b l a_{\mathrm{VIM}-2}$ en 35 cepas, $b l a_{\mathrm{KPC}-2+\mathrm{VIM}-2}$ en 7 cepas y $b l a_{\mathrm{KPC}-2}$ en 2 cepas. En las cepas $b l a_{\mathrm{KPC}-2} \mathrm{se}$ encontró relación clonal entre ellas. Conclusiones: Un $65,3 \%$ de $P$. aeruginosa presentó susceptibilidad disminuida a carbapenémicos, observándose que la presencia de carbapenemasas no es el principal mecanismo de resistencia. Además, se describe la emergencia en Chile de cepas de $P$. aeruginosa con carbapenemasas del tipo KPC-2 sola o en combinación con VIM-2.

Palabras clave: carbapenemasas; resistencia antimicrobiana; Pseudomonas aeruginosa.

\section{Abstract}

Background: Pseudomonas aeruginosa is a relevant infectious agent affecting patients within health care setting; this situation is worsening with the appearance of strains resistance to carbapenems. Aims: To study carbapenemase production in $P$. aeruginosa with decreased susceptibility to carbapenems processed in the microbiology laboratory of the Health Network UC-CHRISTUS in 2014-2015 and compare them with the strains studied in 2004-2005. Methods: Between January 2014 and June 2015, 459 strains of $P$. aeruginosa from clinical samples were isolated. Susceptibility was determined by dilution in agar and strains with reduced susceptibility to carbapenems were tested for carbaNP. Positive strains were studied by PCR for $b l a_{\mathrm{VIM}}$, $b l a_{\mathrm{VIM}-1}, b l a_{\mathrm{VIM}-2}, b l a_{\mathrm{IMP}}, b l a_{\mathrm{NDM}}, b l a_{\mathrm{KPC}}, b l a_{\mathrm{OXA}}$ and $b l a_{\mathrm{IMI}}$ genes. Pulsed field electrophoresis was performed on selected strains. Results: From 459 strains studied, 300 had reduced susceptibility to carbapenems $(65.3 \%)$. Of these, 183 were viable for study, corresponding to 164 patients. The carbaNP test was positive in 44 strains of the 183 strains $(24 \%)$. The resistance genes found were: $b l a_{\mathrm{VIM}-2}$ in 35 strains, $b l a_{\mathrm{KPC}-2+\mathrm{VIM}-2}$ in 7 strains and $b l a_{\mathrm{KPC}-2}$ in 2 strains. In the $b l a_{\mathrm{KPC}-2}$ strains clonal relation between them was found. Conclusions: A $65.3 \%$ of $P$. aeruginosa presented decreased susceptibility to carbapenems being the presence of carbapenemases not the main resistance mechanism. In addition, the emergence in Chile of $P$. aeruginosa strains with bla of the KPC-2 type alone or in combination with VIM-2 is described.

Keywords: carbapenemases; antimicrobial resistance; Pseudomonas aeruginosa.

\section{Correspondencia a: \\ Patricia García Cañete \\ pgarciacan@uc.cl}




\section{Introducción}

$P$ seudomonas aeruginosa es uno de los principales agentes etiológicos de infecciones asociadas a la atención de salud (IAAS), tanto en Chile como mundialmente ${ }^{1}$. En nuestro país, la letalidad asociada se ha calculado para dos patologías: neumonía asociada a ventilación mecánica (NAVM): $12,1 \%$ en neonatos, $25,3 \%$ en adultos y $14,4 \%$ en niños, y para infecciones del torrente sanguíneo asociado a catéter venoso central (ITS CVC): $4 \%$ en neonatos, $12,7 \%$ en adultos y $6,8 \%$ en niños ${ }^{2}$.

En los últimos años se ha observado un aumento en la prevalencia de cepas de $P$. aeruginosa resistentes a carbapenémicos, las que además presentan resistencia múltiple a otros antimicrobianos, dificultando su tratamiento y se asocian a mayor mortalidad ${ }^{3}$.

La resistencia a carbapenémicos puede explicarse por diversos mecanismos que poseen las bacterias; sin embargo, la presencia de carbapenemasas es uno de los más preocupantes por su amplio espectro hidrolítico y por su gran capacidad de diseminación, cuando los genes que las codifican se encuentran contenidos en plásmidos ${ }^{4}$.

En el mundo, la presencia de carbapenemasas en $P$. aeruginosa se ha descrito en Europa, Asia y América siendo la principal las de tipo VIM ${ }^{5,6}$. Sin embargo, desde hace aproximadamente una década se han reportado $P$. aeruginosa productoras de KPC en varios países de Asia y América ${ }^{7,8}$. Las bacterias que poseen $b l a_{\mathrm{KPC}}$ tienen repercusión tanto clínica como epidemiológica; además, se asocian con una mayor resistencia a otros $\beta$-lactámicos y se han descrito en brotes de IAAS 9

En Chile, en el bienio 2004-2005, nuestro grupo detectó por primera vez la presencia de carbapenemasas del tipo VIM en 18,6\% de las cepas de $P$. aeruginosa con susceptibilidad disminuida a imipenem ${ }^{10}$.

El objetivo actual fue estudiar la presencia de carbapenemasas de clase A y B de Ambler en las cepas de $P$. aeruginosa con susceptibilidad disminuida a imipenem y/o meropenem, en el mismo hospital universitario, al cabo de una década de la anterior evaluación.

\section{Métodos}

Estudio retrospectivo de las cepas aisladas de pacientes, entre enero de 2014 y septiembre de 2015, en el Laboratorio de Microbiología del Hospital Clínico de la Universidad Católica.

\section{Métodos microbiológicos}

Las cepas permanecieron almacenadas a $-20^{\circ} \mathrm{C}$ hasta el actual estudio. En caso que un paciente tuviera más de un aislado, se consideró eventos infecciosos diferentes si había más de 30 días entre ambos aislamientos. La iden- tificación de especie de todos los aislados fue confirmada por espectrometría de masas con MALDI-TOFF (Bruker Daltonics, Bremen, Germany).

La susceptibilidad fue determinada por método de dilución en agar para los antimicrobianos con actividad anti-pseudomónica, tales como ceftazidima, cefepime, amikacina, ciprofloxacina, piperacilina/tazobactam, imipenem, meroperem y colistina. Se utilizaron los puntos de corte descritos por el CLSI ${ }^{11}$

\section{Detección de carbapenemasas}

A las cepas con susceptibilidad disminuida a los carbapenémicos se les realizó test carbaNP "in house" según lo descrito por CLSI $2015^{11}$.

\section{Métodos moleculares}

Las cepas con resultado positivo para carbaNP fueron estudiadas genotípicamente por reacción de polimerasa en cadena (RPC) para los genes $b l a_{\mathrm{VIM}}, b l a_{\mathrm{VIM}-1}, b l a_{\mathrm{VIM}-2}$, $b l a_{\mathrm{IMP}}, b l a_{\mathrm{NDM}}, b l a_{\mathrm{KPC}}, b l a_{\mathrm{OXA}}$ y $b l a_{\mathrm{IMI}}$. Los partidores utilizados para cada RPC están especificados en la Tabla 1. Se incluyó en cada RPC un control negativo de reactivos ( $\mathrm{H}_{2} \mathrm{O}$ libre de nucleasas) y un control positivo de ADN de enterobacterias portadoras de los distintos genes de carbapenemasas. El protocolo de amplificación utilizado fue obtenido desde Wozniak y cols ${ }^{12}$. La caracterización del tipo de KPC se realizó por secuenciación utilizando los partidores KPC-up y KPC-dw (Tabla 1) obtenido de Shen y cols. ${ }^{13}$. El producto de RPC obtenido fue purificado y secuenciado mediante el servicio otorgado por Macrogen. Las secuencias fueron analizadas y corregidas utilizando la aplicación online Clustal Omega y el software Chromas id. Posteriormente, las secuencias corregidas fueron alineadas con los distintos alelos de KPC disponibles en la base de datos online GenBank (http://www.ncbi.nlm. nih.gov/genbank/).

\section{Tipificación molecular}

El análisis de clonalidad se realizó exclusivamente en las cepas portadoras de $b l a_{\mathrm{KPC}}$. Se evaluó por método de electroforesis en gel de campo pulsado, usando SpeI como enzima de restricción, según lo descrito por Maslow y Lutsky ${ }^{14}$, interpretada según los criterios de Tenover y $\operatorname{cols}^{15}$.

\section{Análisis estadísticos}

Para comparar la susceptibilidad a antimicrobianos se utilizó el test de $\chi^{2}$, considerando diferencia significativa $\mathrm{p}<0,05$.

\section{Resultados}

De las 459 cepas de $P$. aeruginosa aisladas, 300 de ellas presentaban susceptibilidad disminuida a carbapenémicos 


\begin{tabular}{|c|c|c|c|c|}
\hline & Nombre & Secuencia & Tamaño del producto RPC (pb) & Referencia \\
\hline$b / a_{\mathrm{KPC}}$ & $\begin{array}{l}\text { KPCF } \\
\text { KPC R }\end{array}$ & $\begin{array}{l}5^{\prime} \text {-TGTCACTGTATCGCCGTC-3' } \\
5^{\prime} \text {-CTCAGTGCTCTACAGAAAACC - } 3^{\prime}\end{array}$ & 1010 & 18 \\
\hline$b / a_{\mathrm{IMI}}$ & $\begin{array}{l}\text { IMI-A } \\
\text { IMI-B }\end{array}$ & $\begin{array}{l}5^{\prime} \text {-ATAGCCATCCTTGTTTAGCTC-3’ } \\
5^{\prime} \text {-TCTGCGATTACTTTATCCTC-3`' }\end{array}$ & 818 & 19 \\
\hline$b / a_{\mathrm{VIM}}$ & $\begin{array}{l}\text { VIM S } \\
\text { VIM AS }\end{array}$ & $\begin{array}{l}\text { 5'- CCGATGGTGTTTGGTCGCAT-3' } \\
\text { 5'- GAATGCGCAGCACCAGGAT- 3' }\end{array}$ & 391 & 10 \\
\hline$b / a_{\mathrm{NDM}-1}$ & $\begin{array}{l}\text { NDM1 F } \\
\text { NDM1 R }\end{array}$ & $\begin{array}{l}\text { 5'- CGGGGCAGTCGCTTCCAAGG-3' } \\
\text { 5'- CCAGCCATTGGCGGCGAAAG-3 }\end{array}$ & 295 & 20 \\
\hline$b / a_{\mathrm{IMP}}$ & $\begin{array}{l}\text { IMPS } \\
\text { IMP AS }\end{array}$ & $\begin{array}{l}\text { 5'-AAAGA TACTGAAAAGTTAGT-3' } \\
5^{\prime} \text {-TCYCCAAYTTCACTRTGACT-3' }\end{array}$ & 446 & 10 \\
\hline$b / a_{\text {OXA-48 }}$ & $\begin{array}{l}\text { OXA-48A } \\
\text { OXA-48B }\end{array}$ & $\begin{array}{l}5^{`} \text { - TTGGTGGCATCGATTATCGG-3`' } \\
5^{\prime} \text { - GAGCACTTCTTTTGTGATGGC-3` }\end{array}$ & 744 & 21 \\
\hline$b / a_{\mathrm{VIM}-1}$ & $\begin{array}{l}\text { VIM-G1-F } \\
\text { VIM-G1-R }\end{array}$ & 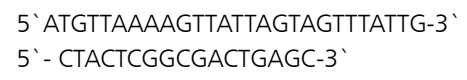 & 801 & 22 \\
\hline$b / a_{\mathrm{VIM}-2}$ & $\begin{array}{l}\text { VIM-G2-F } \\
\text { VIM-G2-R }\end{array}$ & 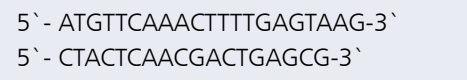 & 801 & 22 \\
\hline$b / a_{\mathrm{KPC}-2}$ & $\begin{array}{l}\text { KPC-Up } \\
\text { KPC-Dw }\end{array}$ & $\begin{array}{l}\text { 5'-GCTACACCTAGCTCCACCTTC-3' } \\
\text { 5'-ACAGTGGTTGGTAATCCATGC-3' }\end{array}$ & 968 & 13 \\
\hline
\end{tabular}

(65,3\%). De éstas, 183 estaban disponibles o viables para estudio, correspondientes a 164 pacientes.

De las 183 muestras estudiadas, 129 (70\%) correspondían a pacientes hospitalizados y $54(30 \%)$ a pacientes ambulatorios. El origen de las muestras fue: respiratorias (n: 70), orina (n: 50), secreción de herida (n: 22), hemocultivos (n: 8), cultivos de punta de catéter venoso central (n: 6), otros sitios (n: 17) y vigilancias (n: 10).

De los 183 aislados estudiados, el test de carbaNP fue positivo en 44 (24\%).

Los resultados de la RPC confirmaron la presencia de carbapenemasas en todas las cepas con test carba NP positivo. Los genes de resistencia encontrados fueron: $b l a_{\mathrm{VIM}-2}$ en 35 cepas y $b l a_{\mathrm{KPC}-2+\mathrm{VIM}-2}$ en siete cepas y $b l a_{\mathrm{KPC}-2}$ en dos cepas. De éstas 79,5\% correspondían a muestras de hospitalizados y 20,5\% ambulatorios. El 40,9\% de las muestras eran de origen respiratorio.

Todos los aislados con $b l a_{\mathrm{KPC}-2}$ provenían de pacientes hospitalizados de muestras respiratorias (n: 3), orinas (n: 2), punta de catéter venoso central (n: 1), secreción de herida operatoria (n: 1), y vigilancias (n: 2). Una de cada cinco cepas $(20 \%)$ de $P$. aeruginosa productoras de carbapenemasas poseen el gen $b l a_{\mathrm{KPC}-2}$, lo que representa $4,9 \%$ del total con susceptibilidad disminuida. De las nueve cepas de $P$. aeruginosa portadores del gen $b l a_{\mathrm{KPC}}$ solo o en asociación con VIM-2 se realizó electroforesis de campo pulsado en siete cepas, resultando todas clonales (Figura 1).
Se comparó el patrón de resistencia de las cepas de $P$. aeruginosa no portadoras de carbapenemasas con aquellas cepas que sí las presentaban (Figura 2), observándose una diferencia estadísticamente significativa para amikacina (36 vs 68\%; $\mathrm{P}=0,01$ ), ceftazidima (58 vs 93\%; $\mathrm{P}=0,005$ ), cefepima $(26 \%$ vs $91 \% ; \mathrm{P}=0,002)$, cefoperazona/sulbac-

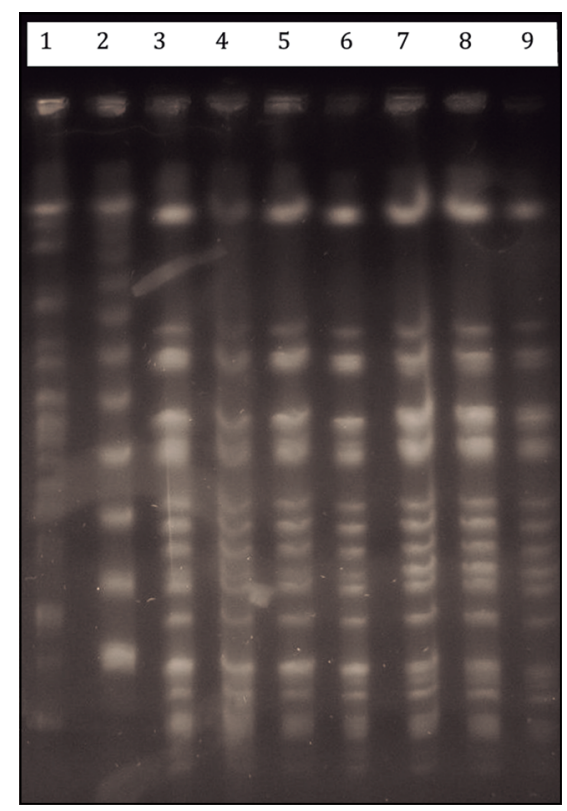

Figura 1. Estudio de clonalidad en cepas de $P$. aeruginosa bla $\mathrm{KPC}-2_{2}$. Carril 1: control enzima restricción. Carril 2: ladder. Carriles 3 al 9, pacientes 1 al 7 . 


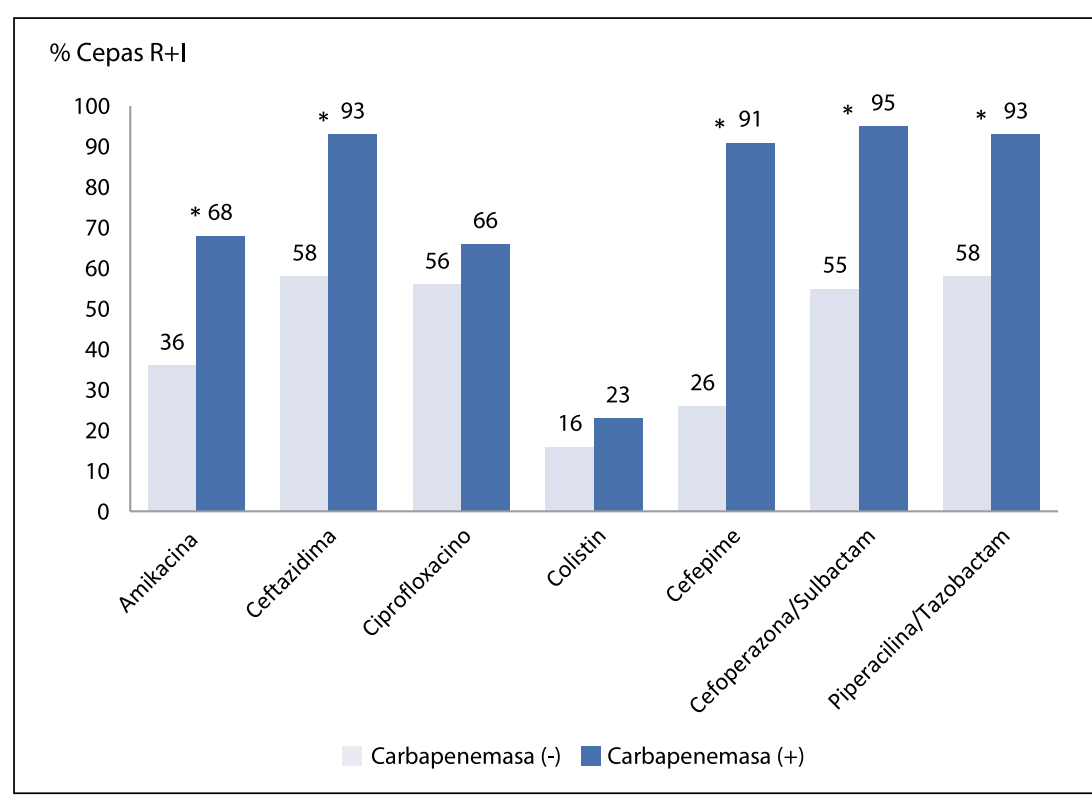

Figura 2. Cepas de $P$. aeruginosa con susceptibilidad disminuida a carbapenémicos. Las cepas con carbapenemasas presentan una mayor resistencia a distintos antimicrobianos estudiados.

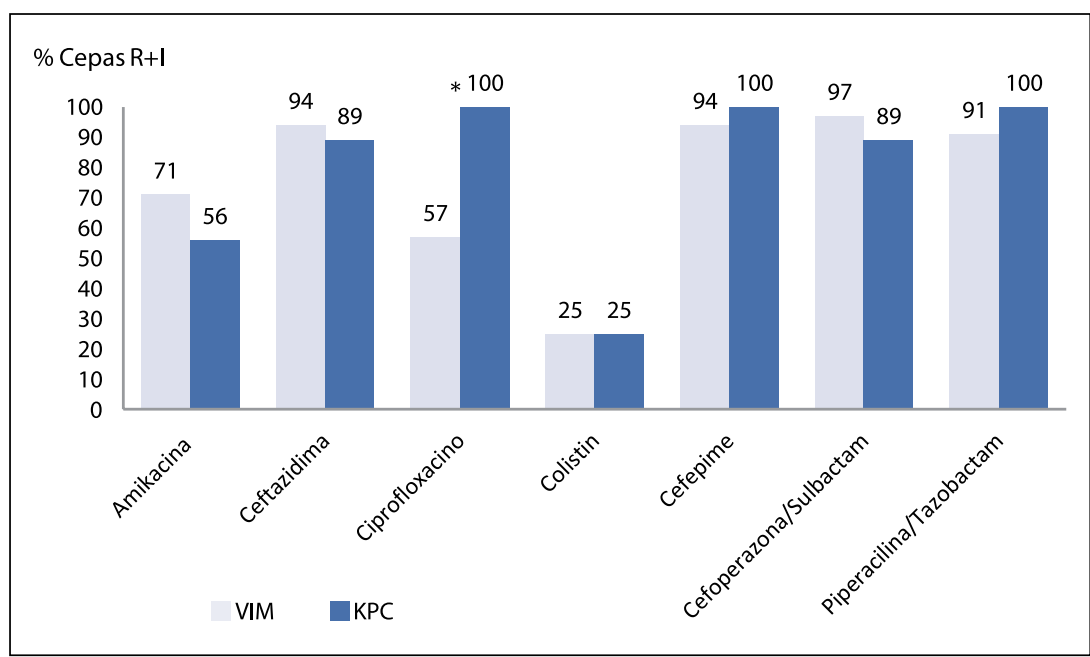

Figura 3. Diferencia de susceptibilidad a distintos antimicrobianos en cepas de $P$. aeruginosa VIM vs KPC. Se muestra que no hubo diferencia significativa para la mayoría de los antimicrobianos, excepto ciprofloxacina en que se observa una resistencia de $57 \%$ en VIM vs $100 \%$ en KPC $(p=0,01)$.

$\operatorname{tam}(55 \%$ vs $95 \% ; \mathrm{P}=0,001)$ y piperacilina/tazobactam (58\% vs 93\%; $\mathrm{P}=0,01)$. No hubo diferencia significativa en ciprofloxacina $(56 \%$ vs $66 \%$; $\mathrm{P}=0,3)$ ni en colistina ( $16 \%$ vs $23 \%$; $\mathrm{P}=0,6)$, destacando que una de cada cinco $P$ aeruginosa con susceptibilidad disminuida a carbapenémicos era también resistente a colistina.

Por último, en la Figura 3 se muestran las diferencias en el patrón de susceptibilidad entre las cepas VIM vs KPC. Nuestros resultados muestran que no hubo diferencia significativa para la mayoría de los antimicrobianos, excepto ciprofloxacina en que se observó una resistencia de $57 \%$ en VIM vs $100 \%$ en KPC $(\mathrm{P}=0,01)$.

\section{Discusión}

Pseudomonas aeruginosa posee múltiples mecanismos de resistencia a carbapenémicos, siendo los más frecuentes la presencia de porinas y la sobre-expresión de bombas de expulsión. Sin embargo, las carbapenemasas son un problema médico y de salud pública, ya que las infecciones producidas por bacterias que poseen carbapenemasas tienen un peor pronóstico para el paciente y limitan el enfrentamiento terapéutico debido a que muy pocos antimicrobianos son eficaces para su manejo ${ }^{16}$.

Datos nacionales describen entre 10 y $30 \%$ de resistencia a carbapenémicos, sin precisar los mecanismos de resistencia asociados ${ }^{17,18}$. En el bienio 2004-2005, nuestro centro reportó $15,3 \%$ de resistencia a imipenem en $P$. aeruginosa, de las cuales $18,6 \%$ presentaban carbapenemasas del tipo VIM $^{10}$. Con la finalidad de complementar los resultados del período 2004-2005, en el presente estudio se incluyó la evaluación de meropenem observando que $65,3 \%$ de las cepas presentaban susceptibilidad disminuida a algún carbapenémico (imipenem o meropenem), lo que representa un aumento significativo y preocupante en la resistencia a estos antimicrobianos. Además, determinamos que este aumento no es explicado por un aumento en las carbapenemasas. Si bien, entre ambos estudios, se observó un aumento porcentual de estas enzimas desde $18,6 \%$ a $24 \%$, este incremento no es significativo, destacándose la aparición de KPC (4,5\%) sola o asociada a VIM (16\%).

Pseudomonas aeruginosa productora de KPC ha sido descrita en Colombia ${ }^{19}$, Brasil ${ }^{20}$, Argentina ${ }^{21}$, E.U.A. ${ }^{22}$ y Puerto Rico ${ }^{23}$, todas del tipo KPC-2; en este último país se ha descrito también KPC-5.

Al evaluar el patrón de susceptibilidad se constató que las cepas $b l a_{\mathrm{KPC}-2} \mathrm{y} b l a_{\mathrm{KPC}-2+\mathrm{VIM}-2}$ tienen resistencia, con una frecuencia que oscila entre $89 \%$ y $100 \%$, a todos los antimicrobianos evaluados, excepto colistina y amikacina.

El hallazgo de $P$. aeruginosa bla ${ }_{\mathrm{KPC}-2}$ sola o en asociación con VIM tiene implicancias terapéuticas y en el control de infecciones. Desde el punto de vista terapéutico, el hecho de que KPC esté asociado a VIM hace que aquellos antimicrobianos muy activos para KPC, pero no para metalo- $\beta$-lactamasa como ceftazidima/avibactam, tengan menos efectividad. A su vez, la capacidad de transmisión de KPC obliga a extremar las medidas de control de infecciones.

El hecho de que siete cepas de $P$. aeruginosa productora de KPC sean clonales, da cuenta de la importante capacidad de diseminación de este agente, lo que ya 


\section{Conclusiones}

aislamientos deben ser cuidadosamente monitorizados para prevenir brotes.

Limitaciones de nuestro trabajo son, por una parte, que reúne información de un sólo centro universitario, por lo que nuestros resultados podrían no ser extrapolables a otros hospitales del país. Por otra parte, los datos de resistencia a colistina podrían estar sobre-estimados ya que el método utilizado en los años en que fue realizado el estudio no se considera en la actualidad el método de referencia ${ }^{25}$.
Nuestro estudio muestra que $65,3 \%$ de las cepas de $P$. aeruginosa presentan susceptibilidad disminuida a algún carbapenémico (imipenem o meropenem) y que, aproximadamente un tercio de ellas, presenta alguna carbapenemasa, especialmente del tipo VIM. Reportamos la presencia de carbapenemasas del tipo KPC en $P$. aeruginosa en nuestro país ya sea sola o asociada a la presencia de VIM.

\section{Referencias bibliográficas}

1.- https://www.minsal.cl/wp-content/ uploads/2018/05/informe-con-indice-yord-2016.pdf.

2.- Informe de Vigilancia de Infecciones Asociadas a Atención en Salud 2017. MINSAL. https:// www.minsal.cl/wp-content/uploads/2015/09/ informe-vigilancia-2017.pdf.

3.- Yu Zhang, Xiao-Li Chen, Ai-Wei Huang, Su-Ling Liu, Wei-Jiang Liu, Ni Zhang, et al. Mortality attributable to carbapenem-resistant Pseudomonas aeruginosa bacteremia: a metaanalysis of cohort studies. Emerg Microbes Infect 2016; 5, e27; doi: 10.1038/emi.2016.22.

4.- Mathers A, Peirano G and Pitout J. The role of epidemic resistance plasmids and international high-risk clones in the spread of multidrugresistant Enterobacteriaceae. Clin Microbiol Rev 2015; 28 (3): 565-91. doi: 10.1128/ CMR.00116-14.

5.- Tsakris A, Poulou A, Kristo I, Pittaras T, Spanakis N, Pournaras S, et al. Large dissemination of VIM-2-metallo- $\beta$-lactamaseproducing Pseudomonas aeruginosa strains causing health care-associated communityonset infections. J Clin Microbiol 2009; 47 (11): 3524-9. doi: 10.1128/JCM.01099-09.

6.- Jing-Jou Yan, Po-Ren Hsueh, Wen-Chien Ko, Kwen-Tay Luh, Shu-Huei Tsai, HsiuMei Wu, et al. Metallo $\beta$-lactamases in clinical Pseudomonas isolates in Taiwan and identification of VIM-3, a novel variant of the VIM-2 enzyme. Antimicrob Agents Chemother 2001; 45(8): 2224-8. doi: 10.1128/ AAC.45.8.2224-2228.2001.

7.- Guevara A, Sierra C, de Waard J. Caracterización molecular de Pseudomonas aeruginosa resistente a carbapenémicos provenientes de cuatro hospitales de Venezuela. Rev Chilena Infectol 2012; 29 (6): 614-21. doi: 10.4067/S0716-10182012000700005.

8.- Vanegas J, Cienfuegos A, Ocampo A, López L, del Corral H, Roncancio G, et al. Similar frequencies of Pseudomonas aeruginosa isolates producing KPC and VIM carbapenemases in diverse genetic clones at tertiary- care hospitals in Medellín, Colombia. J Clin Microbiol 2014; 52 (11): 3978-86. doi: 10.1128/JCM.01879-14.

9.- García D, Nicola F, Zárate S, Relloso S, Smayevsky J, Arduino S. Emergence of Pseudomonas aeruginosa with KPC-type carbapenemase in a teaching hospital: an 8-year study. J Med Microbiol 2013, 62: 1565-70. doi: 10.1099/jmm.0.059923-0.

10.- Pérez A, García P, Poggi H, Braun S, Castillo C, Román J C, et al. Presencia de metalo$\beta$-lactamasas en Pseudomonas aeruginosa resistente a imipenem. Rev Med Chile 2008; 136: 423-32. doi: /S0034-98872008000400002.

11.- Clinical and Laboratory Standards Institute (CLSI). Performance Standards for Antimicrobial Susceptibility Testing, Approved Standard-Eleventh Edition. CLSI document M02-A12; Wayne, PA; 2015.

12.- Wozniak A, Villagra N A, Undabarrena A, Gallardo N, Keller N, Moraga M, et al. Porin alterations present in non-carbapenemaseproducing Enterobacteriaceae with high and intermediate levels of carbapenem resistance in Chile. J Med Microbiol 2012; 61: 1270-9. doi: 10.1099/jmm.0.045799-0.

13.- Shen P, Wei Z, Jiang Y, Xu X, Ji S, Yu Y, et al. Novel genetic environment of the carbapenemhydrolyzing $\beta$-lactamase KPC-2 among Enterobacteriaceae in China. Antimicrob Agents Chemother. 2009; 53: 4333-8. doi: 10.1128/AAC.00260-09.

14.- Maslow J, Slutsky A, Arbeit R. Application of pulsed-field gel electrophoresis to molecular epidemiology. In: Persing D H, Smith T F, Tenover F C, White T J. Diagnostic Molecular Microbiology: Principles and Applications. Washington, DC: American Society for Microbiology 1993: 563-72.

15.- Tenover F, Arbeit R, Goering R, Mickelsen P, Murray B, Persing D, et al. Interpreting chromosomal DNA restriction patterns produced by pulsed-field gel electrophoresis: criteria for bacterial strain typing. J Clin Microbiol 1995; 33: 2233-9. PMID: 7494007.

16.- Falagas M, Tansarli G, Karageorgopoulos D, Vardakas K. Deaths attributable to carbapenemresistant Enterobacteriaceae infections. Emerg Infect Dis 2014; 20(7): 1170-5. doi: 10.3201/ eid2007.121004.

17.- Trucco O, Prado V, Durán C y grupo PRONARES. Red de Vigilancia de Resistencia Antimicrobiana PRONARES. Informe primer semestre 2001. Rev Chilena Infectol 2002; 19 (Supl. 2): S 140-148. http://dx.doi.org/10.4067/ S0716-10182002019200015.

18.- Silva F, Cifuentes M, Pinto M E, en representación del Grupo Colaborativo de Resistencia Antimicrobiana. Resultados de la vigilancia de susceptibilidad antimicrobiana en Chile: Consolidando una red. Rev Chilena Infectol 2011; 28 (1): 19-27. http://dx.doi. org/10.4067/S0716-10182011000100004.

19.- Villegas M B, Lolans K, Correa A, Katann J N, López J A, Quinn J P, et al. First identification of Pseudomonas aeruginosa isolates producing a KPC-type carbapenemhydrolysing $\beta$-lactamase. Antimicrob Agents Chemother 2007; 51: 1553-5. doi: 10.1128/ AAC.01405-06.

20.- Luna P, Rodrigues L, Borges A, Lopes A, Vieira M. First report of KPC-producing Pseudomonas aeruginosa in Brazil. Antimicrob Agents Chemother 2012; 56 (9): 4990. doi: 10.1128/AAC.00699-12.

21.- Santella G, Cittadini R, Papalia M, Vera C, Del Castillo M, Vary C, et al. First clonal spread of KPC-producing Pseudomonas aeruginosa in Buenos Aires, Argentina. Infect Genet Evol 2012; 12: 2003-5. doi: 10.1016/j. meegid.2012.03.022.

22.- Poirel L, Nordmann P, Lagrutta E, Cleary T, Muñoz-Price L. Emergence of KPC-producing Pseudomonas aeruginosa in the United States. Antimicrob Agents Chemother 2010; 54: 3072. doi: 10.1128/AAC.00513-10.

23.- Wolter D J, Khalaf N, Robledo I, Vázquez 
G, Santé M, Aquino E, et al. Surveillance of carbapenem-resistant Pseudomonas aeruginosa isolates from Puerto Rican Medical Center hospitals: dissemination of KPC and IMP-18 $\beta$-lactamases. Antimicrob Agents Chemother 2009; 53(4): 1660-4. doi: 10.1128/AAC.0117208 .
24.- Yan-Yan Hu, Dan-xia Gu, Jia-chang Cai, Hong-wei Zhou, Rong Zhang. Emergence of KPC-2-producing Pseudomonas aeruginosa sequence type 463 isolates in Hangzhou, China. Antimicrob Agents Chemother 2015; 59(5): 2914-7. doi: 10.1128/AAC.04903-14.

25.- Matuschek E, Åhman J, Webster C, Kahlmeter
G. Antimicrobial susceptibility testing of colistin - evaluation of seven commercial MIC products against standard broth microdilution for Escherichia coli, Klebsiella pneumoniae, Pseudomonas aeruginosa, and Acinetobacter spp. Clin Microbiol Infect 2018; 24 (8): 865-70. doi: 10.1016/j.cmi.2017.11.020 